\title{
Efficacy of interferential current on relieving pain of musculoskeletal origin - protocol of a systematic review and meta-analysis undertaken
}

\author{
Hisham Mohamed Hussein ${ }^{1,2, A, C-F \oplus}$, Raghad Alshammari ${ }^{2, A-C, E-F} \oplus$, Sultana Al-Barak ${ }^{2, A-B, D-F} \oplus$, \\ Norah Alshammari ${ }^{2, A-B, E-F} \oplus$, Shahad Alajlan ${ }^{2, A-C, E-F \oplus}$ \\ ${ }^{1}$ Department of Basic Sciences, Faculty of Physical Therapy, Cairo University, Giza, Egypt \\ ${ }^{2}$ Department of Physical Therapy, College of Applied Medical Sciences, University of Hail, Ha'il, Saudi Arabia \\ A - Research concept and design, B - Collection and/or assembly of data, C - Data analysis and interpretation, \\ $D$ - Writing the article, E - Critical revision of the article, F - Final approval of article
}

Hisham Mohamed Hussein, Raghad Alshammari, Sultana Al-Barak, Norah Alshammari, Shahad Alajlan. Efficacy of interferential current on relieving the pain of musculoskeletal origin: a protocol of a systematic review and meta-analysis. J Pre Clin Clin Res. 2020 ; 14(3): 69-72. doi: $10.26444 / j p c c r / 127466$

\begin{abstract}
Introduction and Objective. Interferential current IFC is one of the common electrotherapeutic modalities used in the treatment of painful conditions. Patients with musculoskeletal pain seek medical help in order to reduce their pain that could be achieved using IFC. The current review aims to analyze the recently available information regarding the efficacy of the IFC in alleviating the pain of musculoskeletal origin.

State of knowledge. IFC, as one of the medium frequency currents, has the advantage of being more comfortable and deeply penetrating so that it can reach deeper painful tissues. It has been proposed that IFC can relieve pain through stimulating different body mechanisms, such as the gate mechanism and the release of body opioids. However, the evidence behind the effectiveness of IFC as a pain-relieving modality for musculoskeletal pain has been poorly studied and still not conclusive.

Conclusions. This systematic review will summarize the effects of IFC on relieving musculoskeletal pain as reported through improvement in visual analog scale, numeric pain rating scale, or the McGill pain questionnaire. Through searching multiple databases and including randomized controlled trials published during the last ten years, the findings of the current systematic review and meta-analysis will establish the quality of the recently available evidence and demonstrate if there will be a need for further studies.
\end{abstract}

\section{Key words}

interferential current, pain, musculoskeletal pain, systematic review, protocol

\section{INTRODUCTION}

Pain is one of the major complaints described by patients with musculoskeletal problems. This type of pain originates from an injury or pathology in the structures related to the musculoskeletal system, such as the skeletal muscles, tendons, ligaments, joints, joint capsules, bursae, fascia, and discs $[1,2]$. It demonstrates a high rate of incidence among adolescents, adults, and elderly subjects $[3,4]$. About $53 \%$ of young adults experience this kind of pain at least once in their lifetime. Moreover, $15 \%$ of the population experiences musculoskeletal pain at least once a week [5]. According to the World Health Organization (WHO), up to one-third of the population worldwide live with a painful musculoskeletal condition [6].

Musculoskeletal pain has been linked to various risk factors, e.g. aging, gender, degenerative joint diseases, overwork, bad posture, genetic and psychological factors [7]. Trauma, back pain, and arthritis are the three most common musculoskeletal conditions reported, and for which health

Addres for correspondence: Hisham Mohamed Hussein, Department of Basic Sciences, Faculty of Physical Therapy, Cairo University, Giza, Egypt, Ahmed Elzayat E-mail: drhisham3000@cu.edu.eg

Received:12.08.2020; accepted: 11.09.2020; first published: 15.09.2020 care visits to physicians' offices, emergency departments, and hospitals occur each year [6]. The presence of such pain may negatively affect the physical ability, psychological health, financial status, and lifestyle of the patients. It also places an obvious economic burden on societies and health care insurance facilities [8].

The reduction of musculoskeletal pain occupies a central position among the objectives of most rehabilitation programmes [9]. In the field of physical therapy, musculoskeletal pain has been considered as one of the leading causes for seeking rehabilitation [10]. Local heat [11], ultrasound [12], transcutaneous electrical nerve stimulation [13], and interferential current (IFC) [14-16] are among the commonly used physiotherapeutic modalities for pain relief. IFC could be described as the application of two symmetrical but asynchronous medium frequency currents $(1,000-10,000 \mathrm{~Hz})$ to produce a new low-frequency current (amplitude modulated frequency) at a frequency between $(0-250 \mathrm{~Hz})$.

Being a medium frequency current, IFC meets less skin resistance, has deep penetration, and demonstrate better comfort compared to low-frequency currents [17]. This current has been widely used in different countries worldwide, such as Australia [18], the United Kingdom [19, 20] and Pakistan 
[21]. Different mechanisms have been proposed to explain how IFC could alleviate pain; gate control theory, endogenous opiates [22], improved circulation, and placebo were among these mechanisms [23]. Other authors attributed the painrelieving ability of the IFC to its amplitude modulated frequency characteristics [24].

The current level of evidence regarding the effectiveness of IFC in the treatment of musculoskeletal pain has not been well studied. Two systematic review, conducted in 2010, investigated the efficacy of IFC in treating pain. The first review [25] investigated the efficacy of IFC on muscular pain and included nine RCTs. This review reported a limitation in the number of studies that incorporated IFC as a single treatment, while stating that IFC might be effective if used in addition to other treatment interventions. The second review investigated the analgesic effect of IFC on either the clinical or induced pain [26]. According to the authors of the second review, the evidence of IFC effectiveness in pain management isinadequate, and included only nine randomized trials, eight of which were conducted on artificially-induced pain.

Recently, there have been an increasing number of studies addressing IFC as an experimental or control treatment for painful musculoskeletal conditions [26, 27]. The results of these studies might help to improve the quality of evidence, provide a better understanding of the role of IFC in relieving musculoskeletal pain, and identifying the best parameters that could achieve this effect [28].

\section{OBJECTIVE}

The aim of this review is to assess the efficacy of IFC in treating pain of musculoskeletal origin.

\section{MATERIALS AND METHOD}

The current systematic review protocol was registered on the PROSPERO registry (CRD42020188225) and will follow the PRISMA guidelines for reporting systematic reviews and meta-analyses.

Eligibility criteria. Studies were included if they met the following criteria: publication date - studies published between January 2010 - June 2020 were included in the review.

Study design. The authors included randomized controlled trials (RCTs) in which the selected sample was randomly assigned into two or more arms of intervention.

Participants. Studies that included adult subjects (age $\geq$ 18 years) with painful musculoskeletal problems, such as degenerative joint diseases, myofascial pain, muscular pain, discogenic related problems, were included [25].

Interventions and comparators. The intervention of interest in the current review is IFC. In the included studies, IFC should be used either as a single treatment compared to a sham (placebo) control, or applied as a part of the control or experimental intervention.
Outcomes. Pain intensity was the only outcome of interest. To be included, the study assessed pain intensity using the visual analog scale (VAS), numeric pain rating scale (NRS), orthe McGill pain questionnaire. Studies that investigated post-intervention effects, with or without any period of follow-up, were included.

Timing. The outcomewas assessed twice (before and after intervention) or more. Studies with short-term, intermediate, or long-term follow-up were included.

Setting. There were no restrictions by type of setting.

Language. Only articles reported in the English language were included.

Exclusion criteria. Studies that failed to meet all of the previously mentioned inclusion criteria were excluded from this review.

Information sources. A systematic search, including several electronic bibliographic databases (Scopus, CINAHL, Cochrane Library, Web of Science, Medline, Embase, and EBSCO), was conducted in the period between 7-12 June 2020. A manual search of the reference lists of the included RCTs was conducted after the end of screening for eligibility.

Search strategy. Two authors searched the electronic databases for the RCTs published in the English language. The following keywords were used: interferential; interferential therapy; interferential current; musculoskeletal pain; electrotherapy; electroanalgesia; muscle pain; low back pain; shoulder pain; joint pain; osteoarthritis pain. The search guidelines for each database were followed. The same authors manually searched the references list of the included RCTs.

Study records - Data management. The results of the search process were uploaded to the Rayyan QCRI.org [29], an Internet-based software programme that facilitates collaboration among reviewers during the assessment of studies against the inclusion and exclusion criteria. Before the formal screening process, a calibration exercise was undertaken to pilot and refine the screening procedures, and to familiarize the screening team with the Rayyan software.

The team developed a table using Microsoft Excel 2007 spreadsheet to gather the characteristics of the eligible studies. These characteristics included the author's name, year of publication, the setting, and country of the study, outcome measures, interventions, sample size, and details of IFC parameters, in addition to other data. Review manager software 5.4 (Copenhagen, Denmark: The Nordic Cochrane Centre, The Cochrane Collaboration, 2008) were used to manage the data that could be used to conduct the metaanalysis.

Selection process. Rayyan software was used for assessing the studies against the inclusion criteria. The total research findings were refined to remove duplicates, then two reviewers independently screened the titles and abstracts of the studies against the inclusion criteria to determine the potentially eligible articles. When uncertainty occurred odata was incomplete, the full article was downloaded and 
reviewed. An email was sent to the corresponding author to obtain any missing data. The reviewers decided whether or not the article met the inclusion criteria. Any disagreement as solved by the opinion of a third reviewer. The reasons for exclusion as recorded. The reviewers ere not blind to the journal titles, study authors, or institutions.

Data collection process. Using the prepared excel table, the characteristics of each study were extracted and recorded. The responsible reviewer attended a workshop about the clinical appraisal of clinical trials and performed a pilot data collection process before conducting the main review to be more familiar with the process.

Methodological quality assessment. Seven common scales (Delphi List, PEDro, Maastricht, Maastricht-Amsterdam List, Bizzini, van Tulder, and Jadad) were incorporated to form one list of items that covered five areas: patient selection, blinding, intervention, outcomes, and statistics. This incorporated form was used to overcome the limitations of each individual scale [30]. This method of quality assessment was performed in a previous systematic review [25]. Each criterion was graded on a yes/no basis.

For each item listed on the critical appraisal sheet, a score of 1 was given when the item was included in the article, and a score of 0 was given if the item as not included or the information provided by the authorsas insufficient to make a clear statement. In cases where the study did not consider a particular item, the item was marked as 'not applicable' on the critical appraisal sheet.

The scoring for each study was calculated by dividing the number of items included by the number of applicable items. Finally, each study was graded as having low, moderate, or high methodological quality based on how many items from the critical appraisal were met. The cut-off was determined as follows: $0-0.40$ for low methodological quality, 0.41- 0.70 for moderate methodological quality, and $0.71-1.00$ for high methodological quality [25]

Data Synthesis and Analysis. Studies investigating similar outcomes and interventions in addition to those providing clear quantitative data were grouped, evaluated for heterogeneity, and pooled, if possible. If a grouping of the outcome data was not applicable, descriptive, and qualitative summaries were used.

In the present study, a meta-analysis was performed to quantify the pooled effect of IFC alone or as an additive treatment on the intensity of the musculoskeletal pain compared to a placebo, control, or comparison intervention. Because the pooled effect was based on the results of the VAS, NRS, or McGill pain questionnaire, the mean difference was used to quantify the pooled effect. Review Manager software was used to summarize the effects (pooled mean differences) and constructed forest plots for all comparisons. For this analysis, the $95 \%$ confidence interval was used, and a chisquare test for heterogeneity performed.

In the presence of clinical heterogeneity in the study population or intervention, the Der-Simonian and Laird random-effects model of pooling was be used based on the assumption of the presence of inter-study variability to provide a more conservative estimate of the true effect. If there was relative homogeneity, a fixed-effects model was used to pool data. Review Manager Software 5.4 (Cochrane
Collaboration, Oxford, UK) was used to construct this review. Endnote X7 software was used to manage all the references.

\section{Acknowledgment}

None.

\section{Funding}

Self-funded.

\section{Declaration}

The authors declare they have no competing interest.

\section{REFERENCES}

1. Fernandes RdCP, da Silva Pataro SM, De Carvalho RB, Burdorf A. The concurrence of musculoskeletal pain and associated work-related factors: a cross sectional study. BMC Public Health. 2016; 16: 1-9.

2.Shaker A, Ala'a Abdulrhman Almazzah AH, Asiri MSA, AlQahtani MAM, Ali M, Al-Mteer A, et al. Pattern and determinants of musculoskeletal pain among surgeons in Abha and Khamis Mushet City, Saudi Arabia. IJMDC. 2020; 4: 652-9.

3. Blyth FM, Noguchi N. Chronic musculoskeletal pain and its impact on older people. Best Pract Res Clin Rheumatol. 2017; 31: 160-8.

4. Cimas M, Ayala A, Sanz B, Agulló-Tomás M, Escobar A, Forjaz M. Chronic musculoskeletal pain in European older adults: Cross-national and gender differences. Eur J Pain. 2018; 22: 333-45.

5.Azabagic S, Spahic R, Pranjic N, Mulic M. Epidemiology of musculoskeletal disorders in primary school children in Bosnia and Herzegovina. Mater Sociomed. 2016; 28: 164-7.

6. World Health Organization. Musculoskeletal conditions report. Geneva: WHO. 2019.

7. Cimmino MA, Ferrone C, Cutolo M. Epidemiology of chronic musculoskeletal pain. Best Pract Res Clin Rheumatol. 2011; 25: 173-83.

8. Knittle K, De Gucht V, Maes S. Lifestyle-and behaviour-change interventions in musculoskeletal conditions. Best Pract Res Clin Rheumatol. 2012; 26: 293-304.

9. Carvalho E, Bettger JP, Goode AP. Insurance coverage, costs, and barriers to care for outpatient musculoskeletal therapy and rehabilitation services. N C Med J. 2017; 78: 312-4.

10. Calner T, Isaksson G, Michaelson P. Physiotherapy treatment experiences of persons with persistent musculoskeletal pain: a qualitative study. Physiother Theory Pract. 2019: 1-10.

11. Petrofsky J, Laymon M, Lee H. Local heating of trigger points reduces neck and plantar fascia pain. J Back Musculoskelet Rehabil. 2020; 33: 21-8.

12. Amjad F, Shahid HA, Batool S, Ahmad A, Ahmed I. A Comparison on Efficacy of Transcutaneous Electrical Nerve Stimulation and Therapeutic Ultrasound in Treatment of Myofascial Trigger Points. KMUJ. 2016; 8: 3-6.

13. Sayilir S. The short-term effects of TENS plus therapeutic ultrasound combinations in chronic neck pain. Complement Ther Clin Pract. 2018; 31: 278-81.

14. Nazligul T, Akpinar P, Aktas I, Ozkan FU, Hartevioglu HC. The effect of interferential current therapy on patients with subacromial impingement syndrome: A randomized, double-blind, sham-controlled study. Eur J Phys Rehabil Med. 2018; 54: 351-7.

15. Serafim A, Rabel J, Neves M, Da Silva TS, Bertolini GRF. Evaluation of acute low back pain in women after treatment with interferential current. JPCCR. 2019; 13: 57-60.

16. Brown AM, Zifchock RA, Hillstrom HJ. The effects of limb dominance and fatigue on running biomechanics. Gait Posture. 2014; 39: 915-9.

17. Palmer S, Martin D. Interferential current for pain control. UWE Bristol. 2002; 11: 287-300.

18. Chipchase LS, Williams MT, Robertson VJ. A national study of the availability and use of electrophysical agents by Australian physiotherapists. Physiother Theory Pract. 2009; 25: 279-96.

19. Ghulam Sarwar Shah S, Farrow A, Esnouf A. Availability and use of electrotherapy devices: a survey. Int J Ther Rehabil. 2007; 14: 260-4.

20. Tabasam G, Johnson MI. The use of interferential therapy for pain management by physiotherapists. Int J Ther Rehabil. 2006; 13: 357-64 
21. Haq K. Preference of modalities and exercises by physiotherapists. Rawal Medical Journal. 2019; 44.

22. De Domenico G. Pain relief with interferential therapy. Aust J Physiother. 1982; 28: 14-8.

23. Goats G. Interferential current therapy. Br J Sports Med. 1990; 24: 87-92.

24. Johnson MI, Tabasam G. A single-blind investigation into the hypoalgesic effects of different swing patterns of interferential currents on cold-induced pain in healthy volunteers. Arch Phys Med Rehabil. 2003; 84: 350-7.

25. Fuentes JP, Armijo Olivo S, Magee DJ, Gross DP. Effectiveness of interferential current therapy in the management of musculoskeletal pain: a systematic review and meta-analysis. Phys Ther. 2010; 90: 1219-38.

26. Beatti A, Rayner A, Souvlis T, Chipchase L. The analgesic effect of interferential therapy on clinical and experimentally induced pain. Phys Ther Rev. 2010; 15: 243-52.
27. Almeida CC, Azevedo KP, Cacho TA, Leal JBG, Montanini GN, Silva DNT, et al. Effects of electroanalgesia on knee osteoarthritis: study protocol for a randomized, triple-blind, placebo-controlled trial. Clin Trials Degener Dis. 2019; 4: 70-5.

28. de Almeida CC, da Silva VZM, Júnior GC, Liebano RE, Durigan JLQ. Transcutaneous electrical nerve stimulation and interferential current demonstrate similar effects in relieving acute and chronic pain: a systematic review with meta-analysis. Braz J Phys Ther. 2018; 22: 347-54. 29. Ouzzani M, Hammady H, Fedorowicz Z, Elmagarmid A. Rayyan - a web and mobile app for systematic reviews. Syst Rev. 2016; 5.

30. Olivo SA, Macedo LG, Gadotti IC, Fuentes J, Stanton T, Magee DJ. Scales to assess the quality of randomized controlled trials: a systematic review. Phys Ther. 2008; 88: 156-75. 